\title{
Success Rate of Sarem Protocol for Assisted Reproductive Techniques in Patients with Poor Ovarian Response
}

\section{ART I C L E I N F O}

\section{Article Type}

Descriptive Study

\section{Authors}

Roustaei M.* $M D$,

Lahouti A. ${ }^{1} M D$,

Shami M. ${ }^{1} B S C$

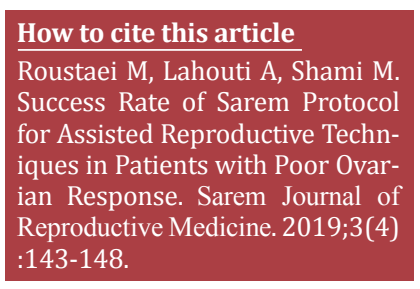

How to cite this article Roustaei M, Lahouti A, Shami M. Success Rate of Sarem Protocol iques in Patients with Poor Ovarian Response. Sarem Journal of :143-148

\begin{abstract}
A B S T R A C T
Aims One of the new challenges in the field of infertility in women is delaying pregnancy due to inadequate response of ovaries to ovulation induction drugs. Many studies have been conducted to provide therapeutic protocols in this regard, but there is not still sufficient and conclusive evidence to introduce a single treatment protocol. The aim of this study was to evaluate the success rate of Sarem Hospital protocol in patients with poor ovarian response to ART.

Instrument \& Methods This study is a descriptive cross-sectional study that was performed on 104 records in Sarem Hospital from 2015 to 2017 that was proved their poor ovarian response according to world criteria and were treated by Sarem Hospital protocol. Data were analyzed by SPSS software and Pearson correlation test.

Findings Of 104 patients, $58(55.8 \%)$ had infertility treatment and had a history of previous IVF and 46 patients (44.2\%) did not receive any specific treatment. The mean duration of infertility was $5.11 \pm 4.67$ years. The mean number of oocytes obtained from the current cycle was $4.79 \pm 2.98$, which was 3.87 more than the number of oocytes of the previous cycle. The mean number of embryos formed was $2.69 \pm 1.75$ and the number of transferred embryos was $2.10 \pm 0.96$. Finally, 21 patients had positive $\beta H C G$ test results and sonography showed FHR+ after three weeks.

Conclusion The protocol approved in Sarem Hospital for patients with poor ovarian response to ART has the potential of $20.2 \%$ successful pregnancy.
\end{abstract}

Keywords Infertility; Poor Ovarian Response; Assisted Reproductive Techniques
*Sarem Fertility \& Infertility Research Center (SAFIR), Iran University of Medical Sciences, Tehran, Iran ${ }^{1}$ Sarem Fertility \& Infertility Research Center (SAFIR), Iran University of Medical Sciences, Tehran, Iran

\section{*Correspondence}

Address: Sarem Women Hospital, Basij Square, Phase 3, Ekbatan Town, Tehran, Iran. Postal Code: 1396956111

Phone: +98 (21) 44670888

Fax: +98 (21) 44670432

masum.rusta58@gmail.com

\section{Article History}

Received: December 15, 2018

Accepted: July 25, 2019

ePublished: October 15, 2019

\section{I T A T I O N L INKS}

[1] Textbook of assisted reproductive technologies: Laboratory and clinical perspectives [2] Poor ovarian reserve [3] Decreased fertility in poor responder women is not related to oocyte morphological status [4] Obesity alters retrieved oocyte count and clinical pregnancy rates in high and poor responder women after in vitro fertilization [5] Human menopausal gonadotropin/human chorionic gonadotropin follicular maturation for oocyte aspiration: Phase II, 1981 [6] Evaluation of the results of ovulation induction with GnRH antagonist (Cetrotide) in patients with poor ovarian response in the previous ART cycle [7] Diminished ovarian reserve, causes, assessment and management [8] ESHRE consensus on the definition of 'poor response'to ovarian stimulation for in vitro fertilization: The bologna criteria [9] Short gonadotropin-releasing hormone agonist versus flexible antagonist versus clomiphene citrate regimens in poor responders undergoing in vitro fertilization: A randomized controlled trial [10] Management of poor responders in IVF: Is there anything new [11] Comparison of different stimulation protocols used in in vitro fertilization: A review [12] Strategies for poor responders in IVF cycles [13] How to define, diagnose and treat poor responders? Responses from a worldwide survey of IVF clinics. Reproductive Biomed Online [14] A new more detailed stratification of low responders to ovarian stimulation: from a poor ovarian response to a low prognosis concept [15] The novel poseidon stratification of 'low prognosis patients in assisted reproductive technology' and its proposed marker of successful outcome [16] Effect of maternal age on the ovarian reserve markers, and pregnancy outcome in a sample of Kurdish women in Erbil city [17] Is the ovarian reserve influenced by vitamin D deficiency and the dress code in an infertile Iranian population? [18] Complete oocyte maturation arrest in art cycles [19] Relation between AMH level and ovarian reserve in infertile women candidate for assisted reproductive treatment [20] Treatment modalities in poor responder patients undergoing assisted reproductive techniques 


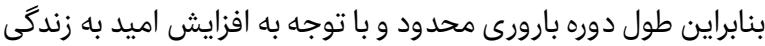

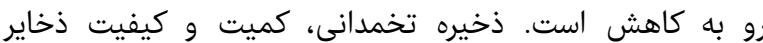

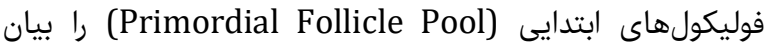

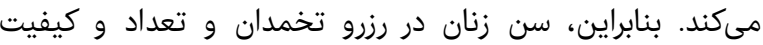

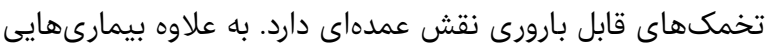

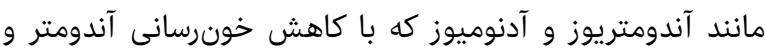

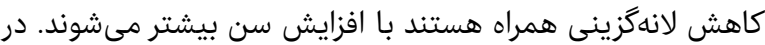

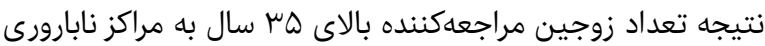

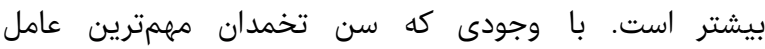

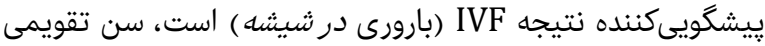

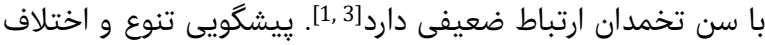

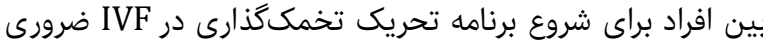

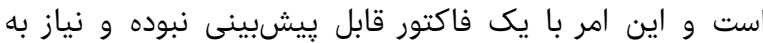

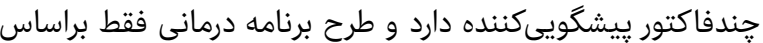

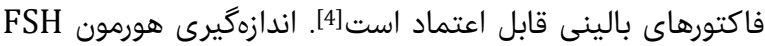

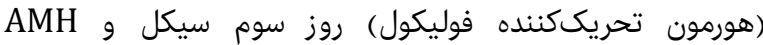

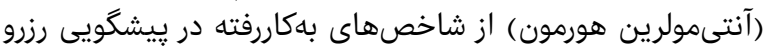

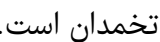

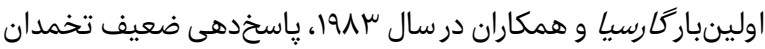

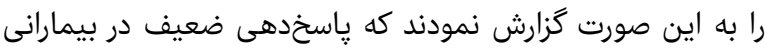

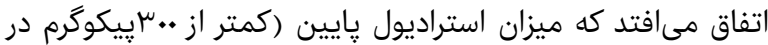

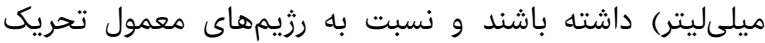

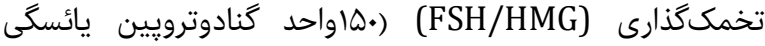
انسانى - HMG باسخ ضعيفى را نشان دهند كه در نتيجه آن، تعداد

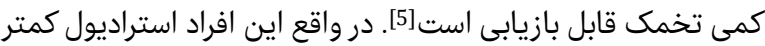

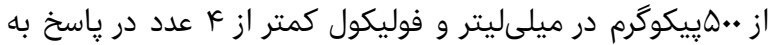

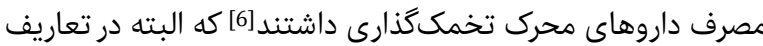

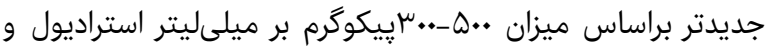

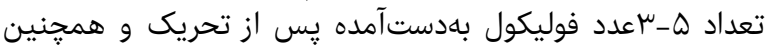

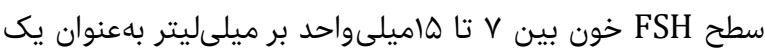

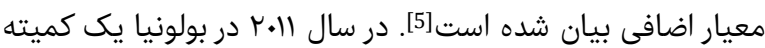

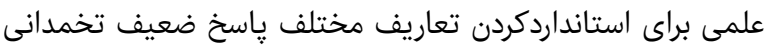

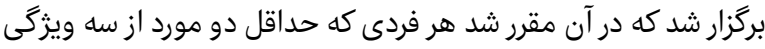

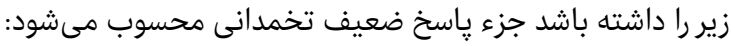

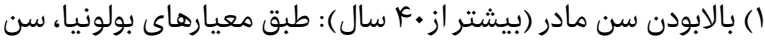

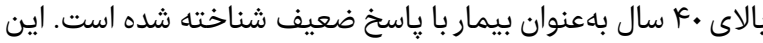

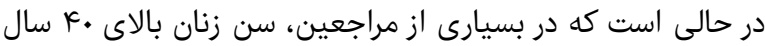

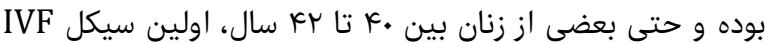

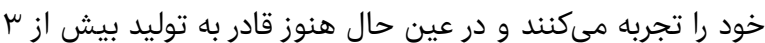

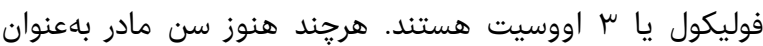
بهترين عامل بيشكويى منفرد حاملكى (Ongoing Pregnancy)

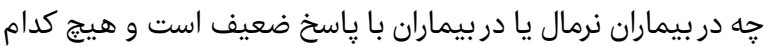

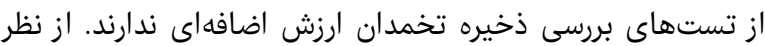

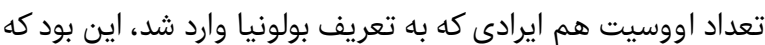

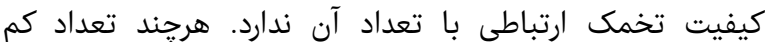

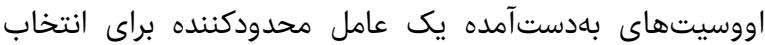

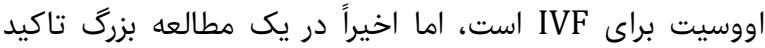

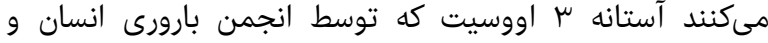

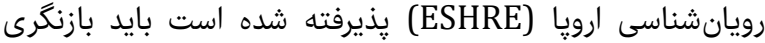

$$
\text { شود ا7ن. }
$$

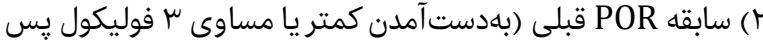

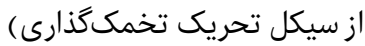

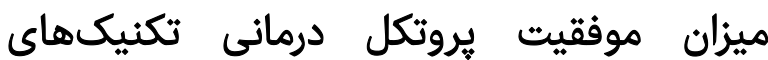 كمكبارورى صارم در بيماران با پاسخ ضعيف درمان

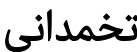

MD " معصومه روستايى

مركز تحقيقات بارورى و نابارورى صارم، دانشكاه علوم يزشكى ايران، تهران، ايران

آمنه لاهوتى تركيقات باروي

مركز تحقيقات بارورى و نابارورى صارم، دانشكاه علوم يزشكى ايران، تهران، ايران

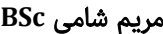

مركز تحقيقات بارورى و نابارورى صارم، دانشگاه علوم يزشكى ايران، تهران، ايران

جكيده

اهداف: يكى از جالشهائ جالش جديد در حوزه نابارورى زنان، بهتاخيرافتادن باردارى

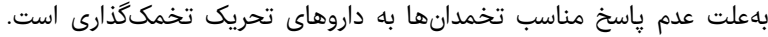

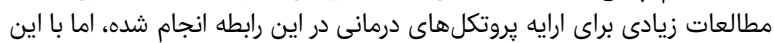

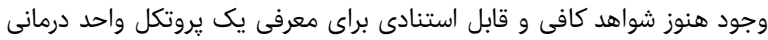

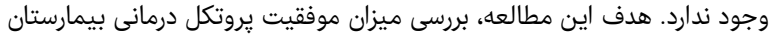

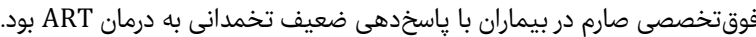

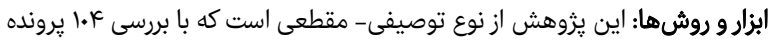

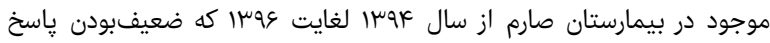

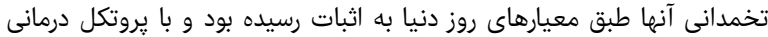

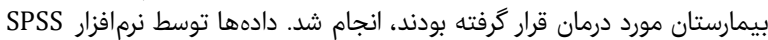

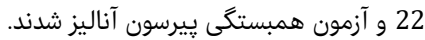

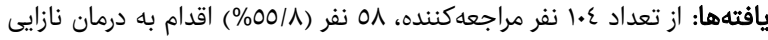

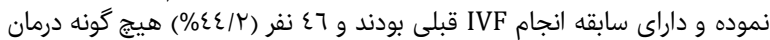

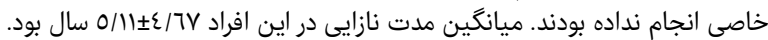

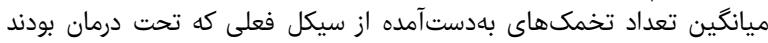

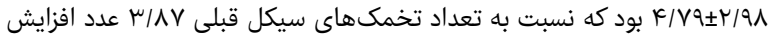

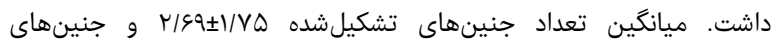

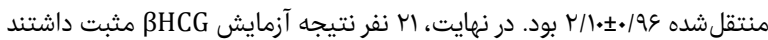

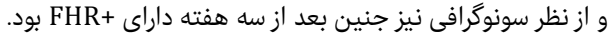

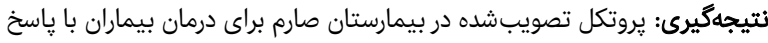

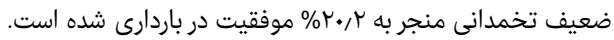
كليدوازوها: نابارورى، ياسخ ضعيف تخمدانى، درمان ART

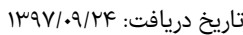

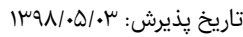

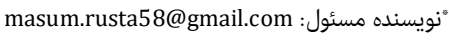

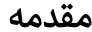

نابارورى حدود •ا تا ها \% زوجين را دركير مى سازد و تعداد افرادى كه

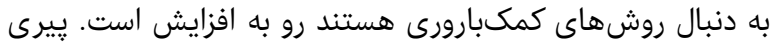

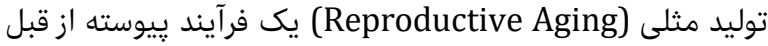

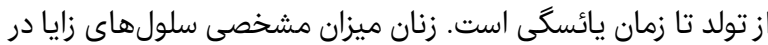

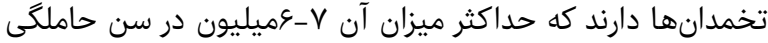

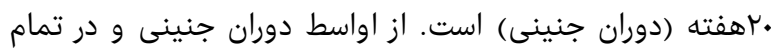

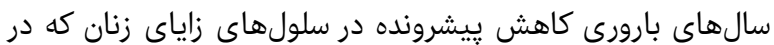

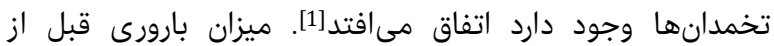

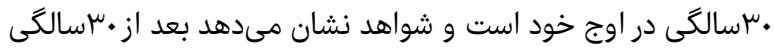

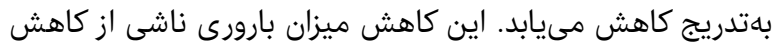

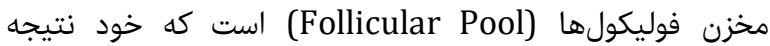

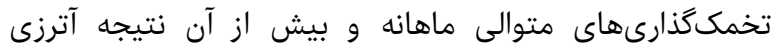

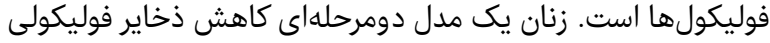

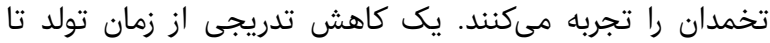

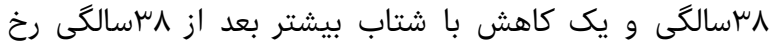
مى دهد. البته ذخيره تخمدانى فوليكولها (Ovarian Reserve)

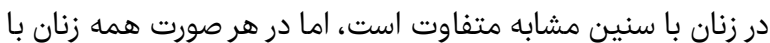

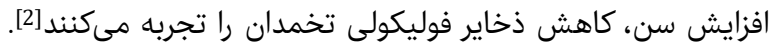




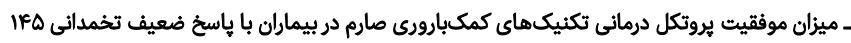

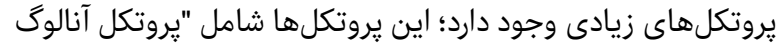
GnRH

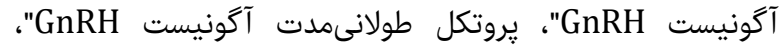

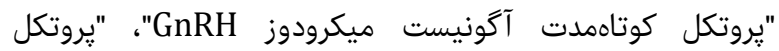
كوتاهدت آثونيست GnRH" و "يروتكل آنتاكونيست GnRH"

مى شوند [11, 12].

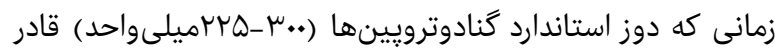

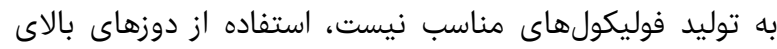

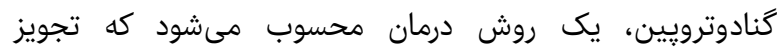

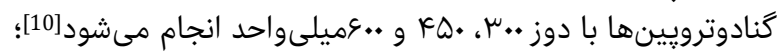

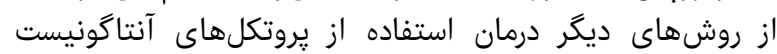

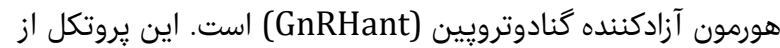

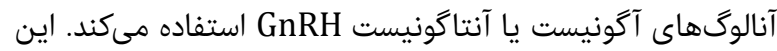

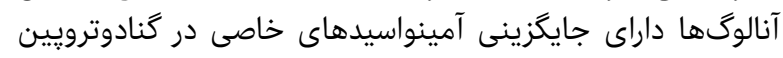

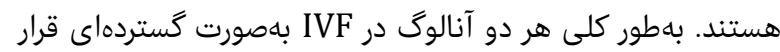

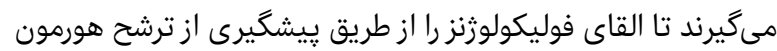
LH

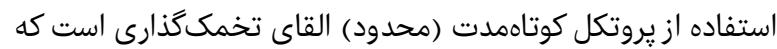

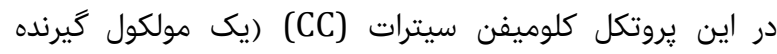

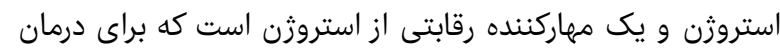

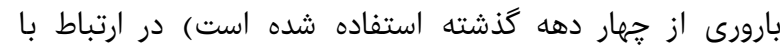

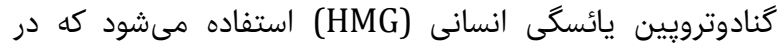

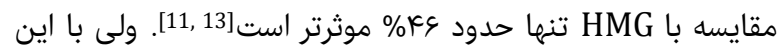

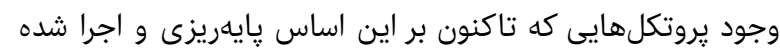

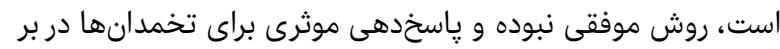

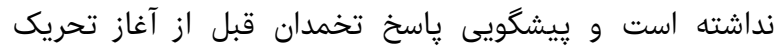

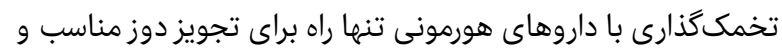

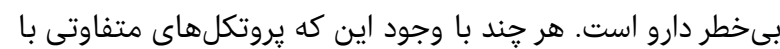

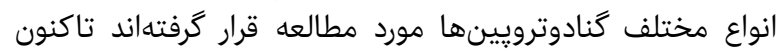

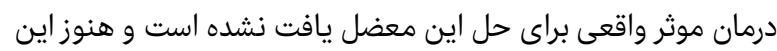

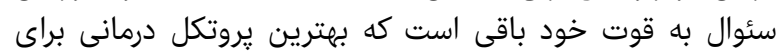

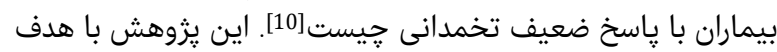

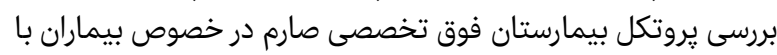
ياسخ ضعيف تخمدانى و نتايج آن انجام شدون

\section{ابزار و روشها}

اين يزوهش از نوع توصيفى - مقطعى است كه با بررسى يروندههاى

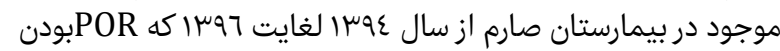

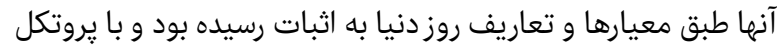

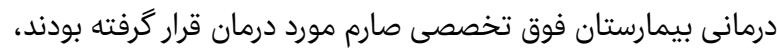

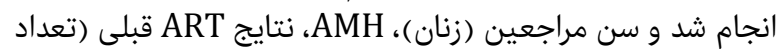

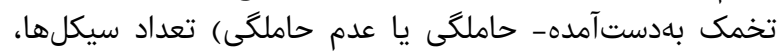

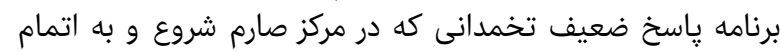

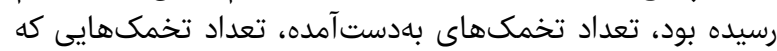

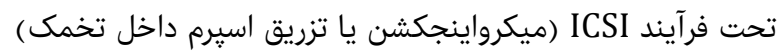

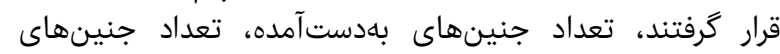

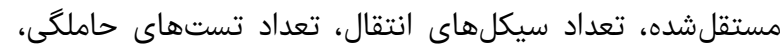

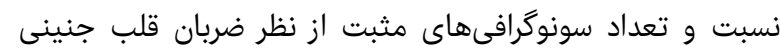

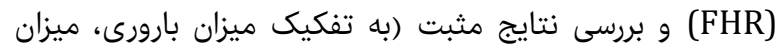

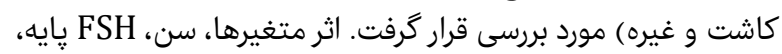

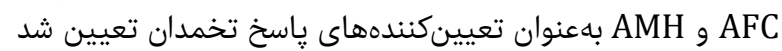

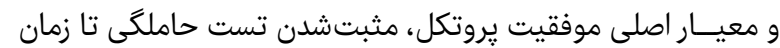

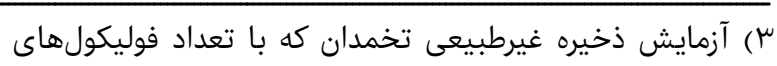

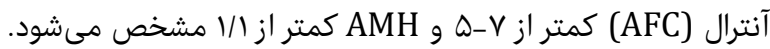

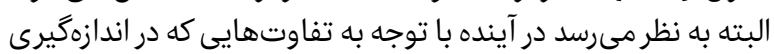

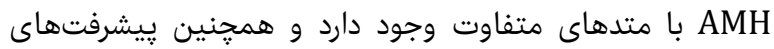

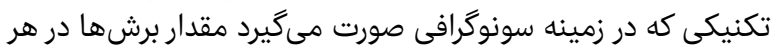

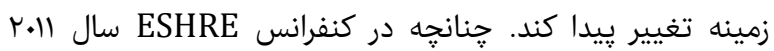

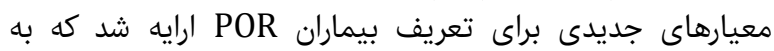

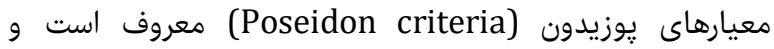

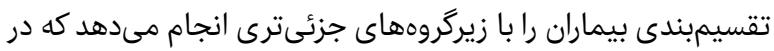

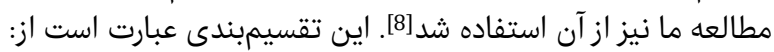

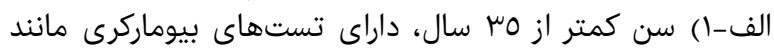

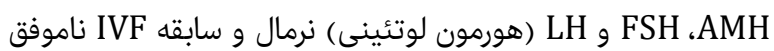

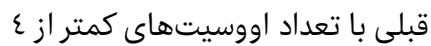

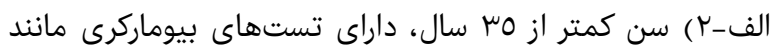

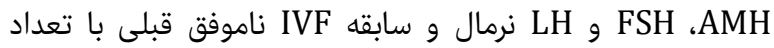

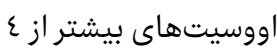

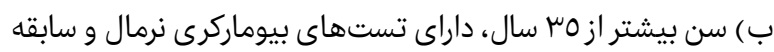
IVF

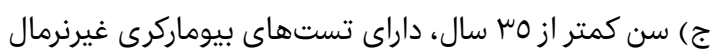

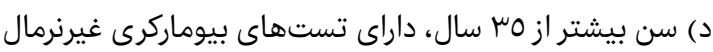

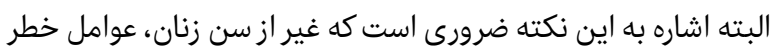

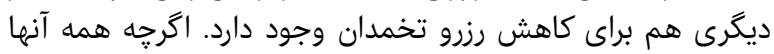

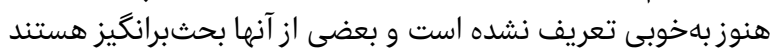

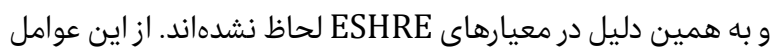

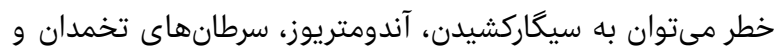

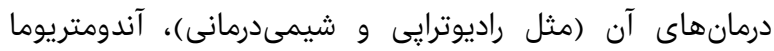

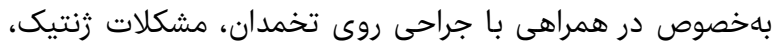

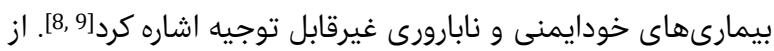

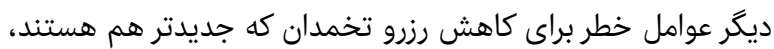

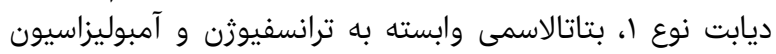

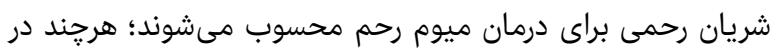

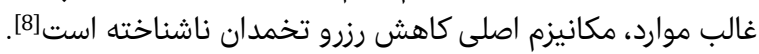

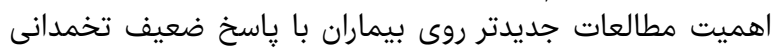

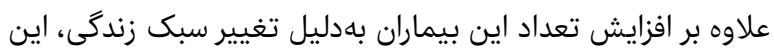

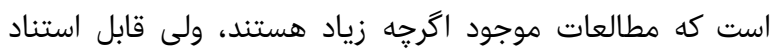

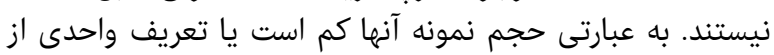

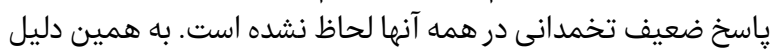

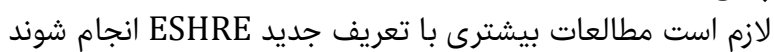

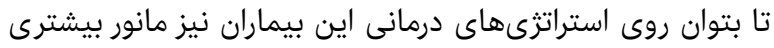

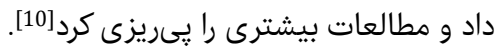

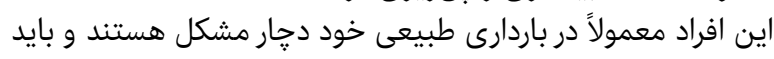

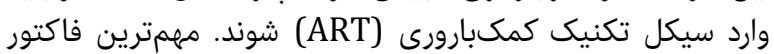

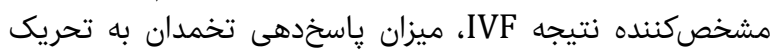

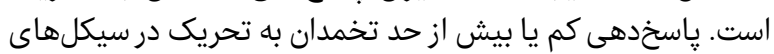

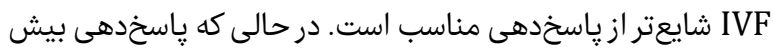

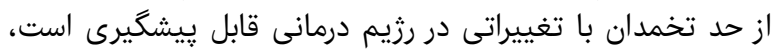

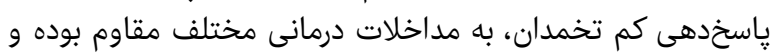

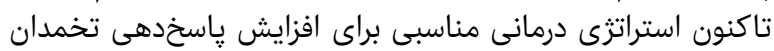

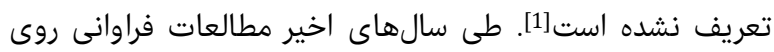

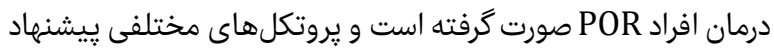

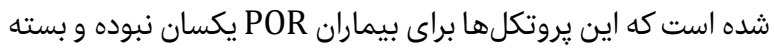

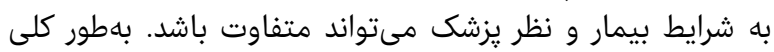




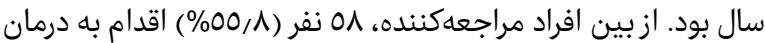

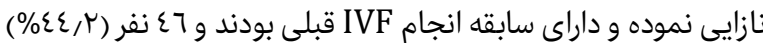
هيج گونه درمان خاصى را انجام نداده بوان بودند.

جدول ا) توزيع فراوانى مطلق و نسبى اطلاعات دموگرافيك بيماران تحت درمان

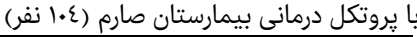

\begin{tabular}{|c|c|c|}
\hline درصد & تعداد & متغيرهاى دموگرافيك \\
\hline$\varepsilon / \wedge$ & 0 & زير •س سال \\
\hline $1 / / \mu$ & 19 & بين •• تا مس سال \\
\hline$\mu 7 / 0$ & щ & بين هب تا •ع سال \\
\hline$\varepsilon \cdot / \varepsilon$ & kr & بالاى •ع سال \\
\hline & & محل سكونت \\
\hline $00 / 1$ & $0 \wedge$ & تهران \\
\hline$V / V$ & $\wedge$ & استان هاى شمالى كشور \\
\hline$r \varepsilon /$. & ro & استانهاى غربى كشور \\
\hline س/^ & $\varepsilon$ & استانهاى شرقى كشور \\
\hline $0 / 1$ & 7 & استانهاى جنوبى كشور \\
\hline$r / 9$ & $\mu$ & استانهاى مركزى كشور \\
\hline & & سطح تحصيلات \\
\hline$\mu / \Lambda$ & $\varepsilon$ & بىسواد \\
\hline $1 \varepsilon / \varepsilon$ & 10 & سيكل \\
\hline HT/I & $r \mu$ & دييلم \\
\hline$\varepsilon 0 / r$ & $\varepsilon V$ & ليسانس \\
\hline $1 . / 7$ & 11 & فوق ليسانس \\
\hline w/A & $\varepsilon$ & دكترى \\
\hline & & شغل \\
\hline $1 / 9$ & $r$ & خدماتى \\
\hline$r \cdot / r$ & r & ادارى \\
\hline$O r / 9$ & 00 & خانهدار \\
\hline r/凡 & $\varepsilon$ & آزاد \\
\hline$r / / r$ & rr & ساير \\
\hline
\end{tabular}

ميانگين سطح سرمى AMH (آنتىمولرين هورمون) در اين افراد

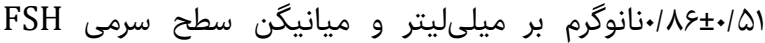

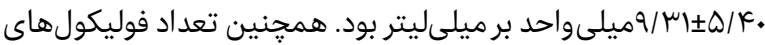

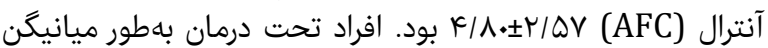

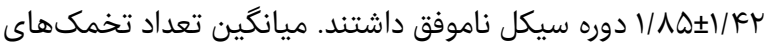

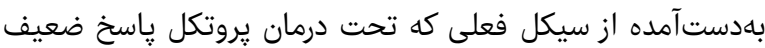

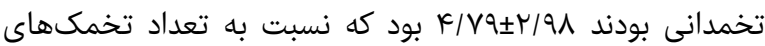

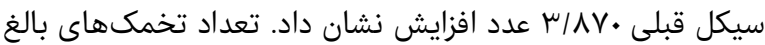

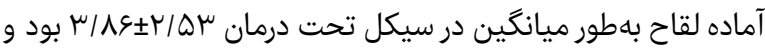

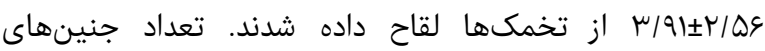

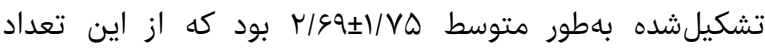

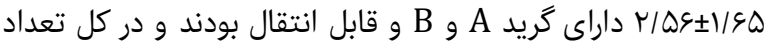

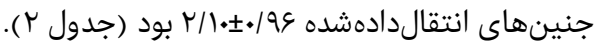

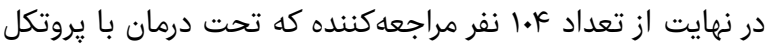

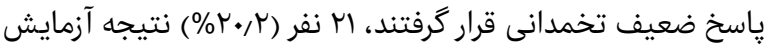
مHCG

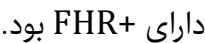
بين تعداد تخمكهاى لقاح دادهشده و مثبتشدن نتيجه تود تست

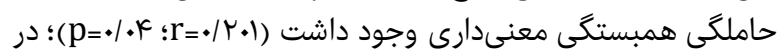

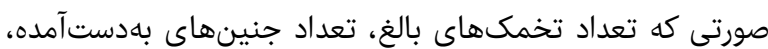

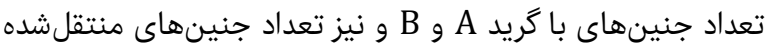

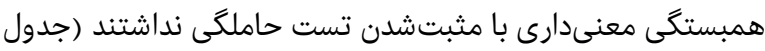

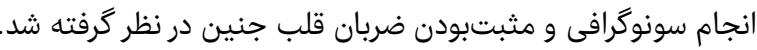

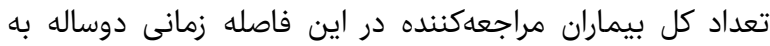

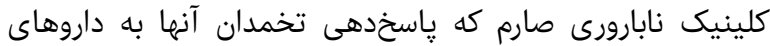
تحريك تخمكگذارى ضعيف بوده و طبق تعاريف و استانداردهاى

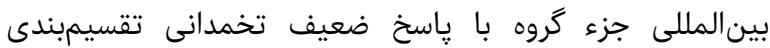
مى شوند، ع.1 نفر بود. طبق جديدترين مقالات روز رون دنيا [14, 15، زنان مراجعهكننده به

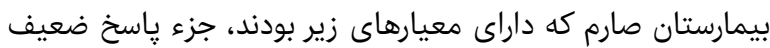
تخمدانى محسوب شدند:

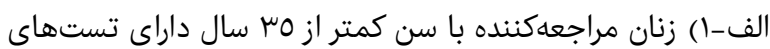

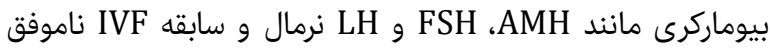
قبلى با ميزان اووسيتهاري ماند كمتر از ع اووسيت

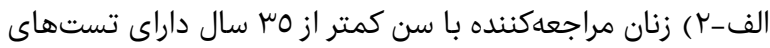

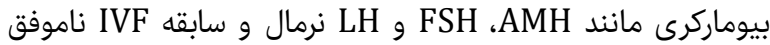
قبلى با ميزان اووسيتهاندان بيشتر از ع اووسيت

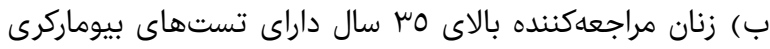

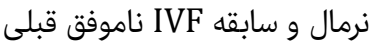
ج) زنان مراجعهكننده با سن كمتر از مس سال دارف داراى تستهاى بيوماركرى غيرنرمال

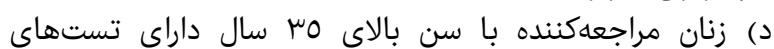
بيوماركرى غيرنرمال

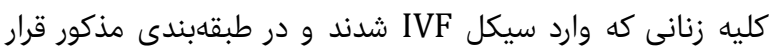

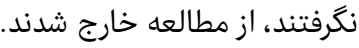

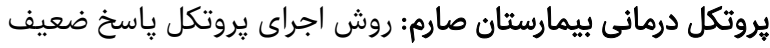

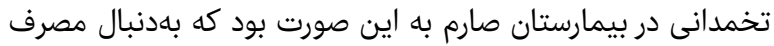

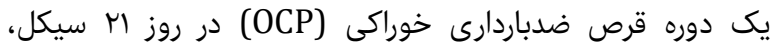

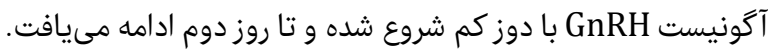

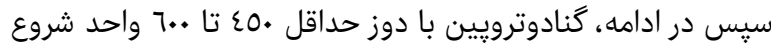

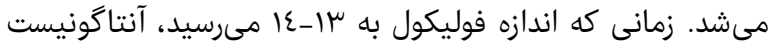

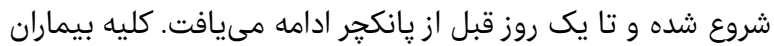

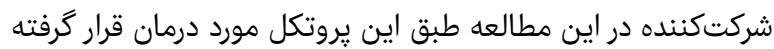

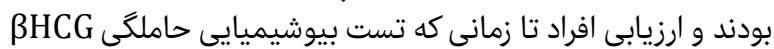

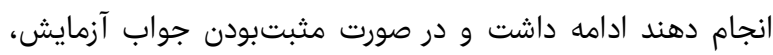

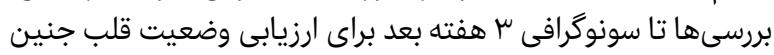

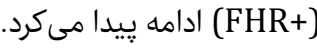

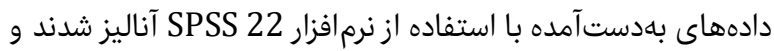

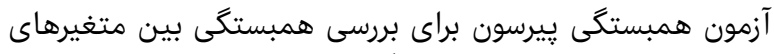

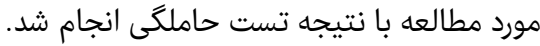

\section{يافتهها}

از بين عـا بيمار مراجعهكننده در فاصله زمانى دوساله به كلينيك

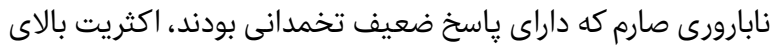

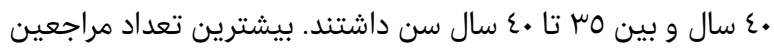

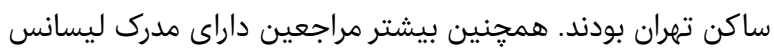

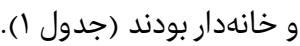

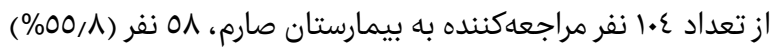

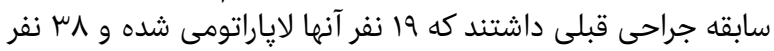

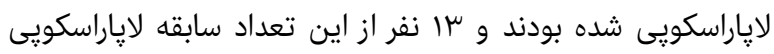

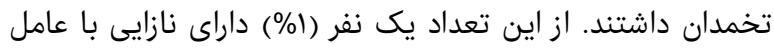

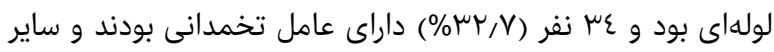

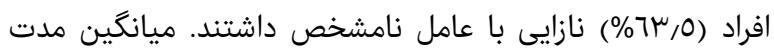

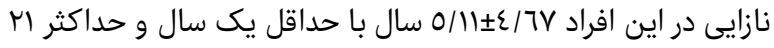




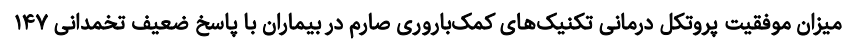

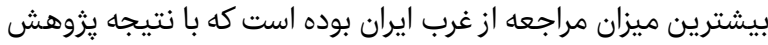

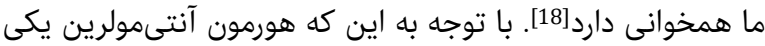

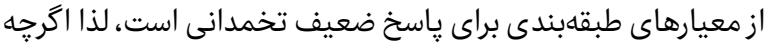

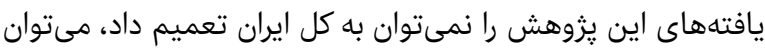

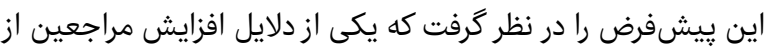

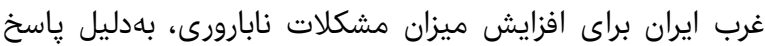
ضعيف تخمدانى است.

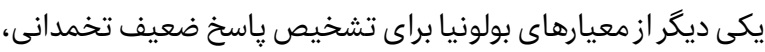

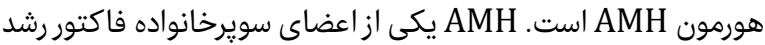

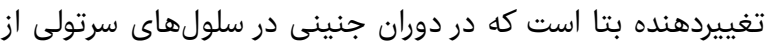

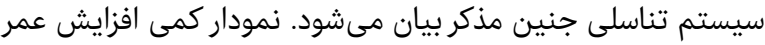

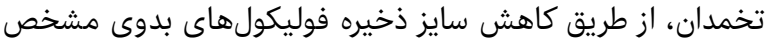

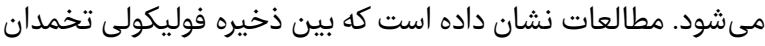
و سطح سرمى AMH يك رابطه مثبت وجود دارد. اين هورمون

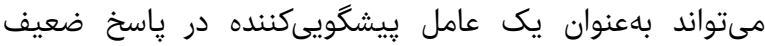

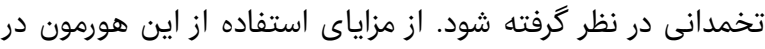

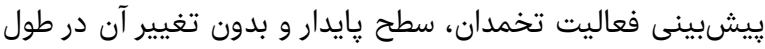

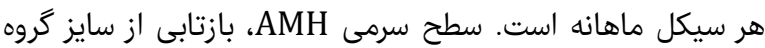

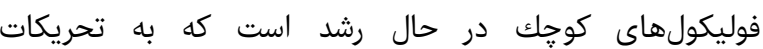
كنادوترويينها بسيار حساس هستند [19]. سطح هورمونى

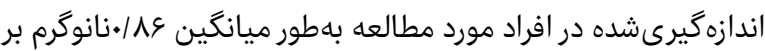

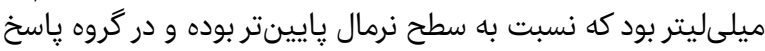

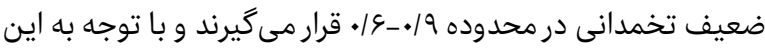

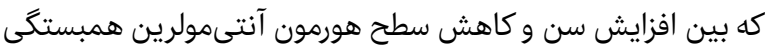

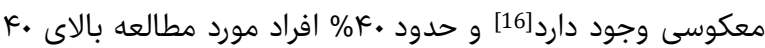

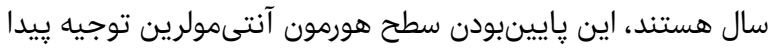

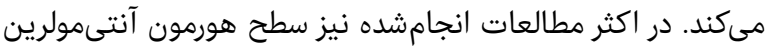

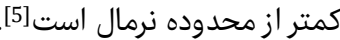
تعداد فوليكولهاى آنترال بهدستآ آمده كه يكى ديكر از معيارهاي

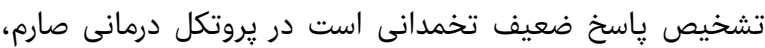
F/A.

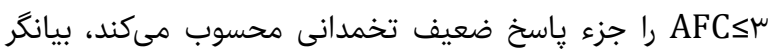

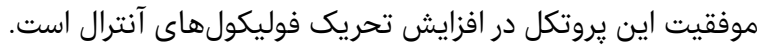

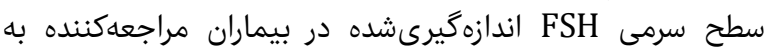

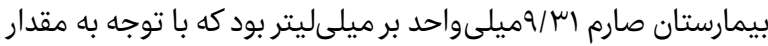

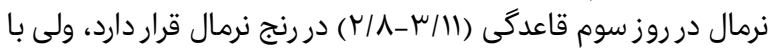

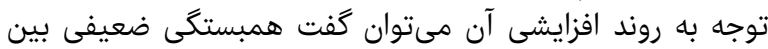

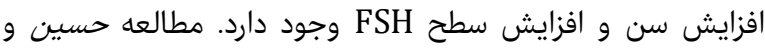

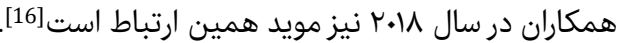

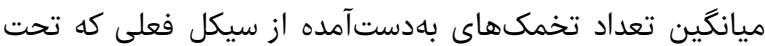

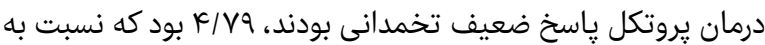

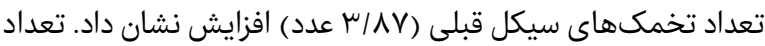

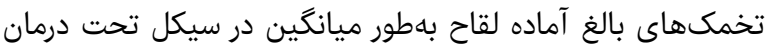

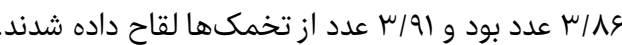

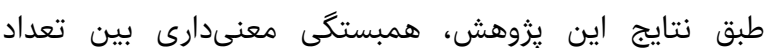

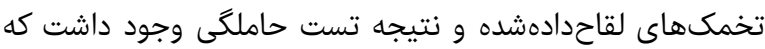

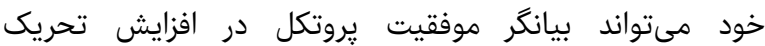

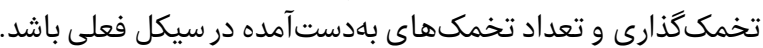

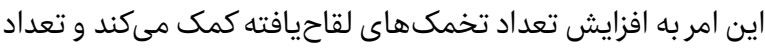

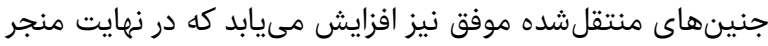

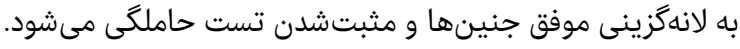

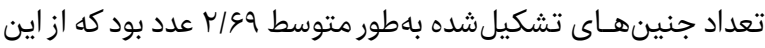

جدول r) ميانكين متغيرهاى مورد مطالعه در افراد تحت درمان با يروتكل پاسخ

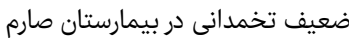

\begin{tabular}{|c|c|}
\hline ميانكين آمارى & متغيرها \\
\hline $0 / 11 \pm \varepsilon / 7 V$ & مدت نازايى (سال) \\
\hline$\varepsilon / \vee १ \pm r / १ \Lambda$ & تعداد تخمكىهاى بهدست آمده در اين سيكل \\
\hline$\cdot / 17 \pm \cdot / 01$ & (نانوكرم بر ميلىليتر) AMH \\
\hline$\varepsilon / \Lambda \cdot \pm r / 0 V$ & تعداد فوليكولهاى آنترال (AFC) \\
\hline$q / \mu / \pm 0 / \varepsilon$. & FSH (ميلىواحد بر ميلىليتر) \\
\hline$r / \Lambda V \pm r / 7 r$ & تعداد تخمكها در سيكل قبلى \\
\hline $1 / 10 \pm 1 / \varepsilon r$ & تعداد سيكلهاى ناموفق قبلى \\
\hline$\varepsilon / V\urcorner \pm r / 9 \vee$ & تعداد تخمكنها \\
\hline$r / \Lambda 7 \pm r / O r$ & تعداد تخمكهاى بالغ \\
\hline$r / 91 \pm r / 07$ & تعداد تخمكنهاى لقاحدادهده \\
\hline$r / 79 \pm 1 / V_{0}$ & تعداد جنين \\
\hline$r / 07 \pm 1 / 70$ & تعدادجنينها با كريد A و B \\
\hline$r / 1 \cdot \pm \bullet / 97$ & تعداد جنينهاى منتقلشده \\
\hline
\end{tabular}

\begin{tabular}{|c|c|c|}
\hline سطح معنى دارى & ضريب همبستكى & متغيرها \\
\hline $.1 . \varepsilon$ & $\cdot / r \cdot 1$ & تعداد تخمك لقاح دادهشده \\
\hline •/乏乏० & $.1 \cdot V 7$ & تعداد تخمك بالغ \\
\hline - / 4 А9 & $.11 \cdot 0$ & تعداد جنين \\
\hline • & $\cdot / 1 \cdot r$ & تعداد جنين باكريد A و B \\
\hline $.10 \mathrm{~V} \varepsilon$ & .1 .07 & تعداد جنين منتقلشده \\
\hline
\end{tabular}

بحث

اين يزروهش با هدف بررسى ميزان موفقيت (ميزان باردارى مثبت)

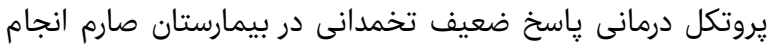

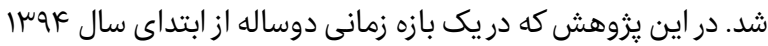

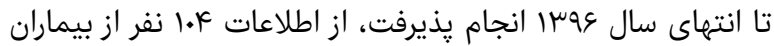

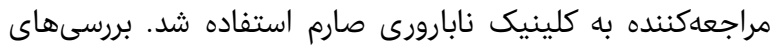

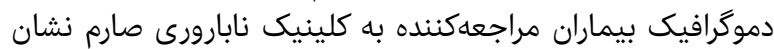

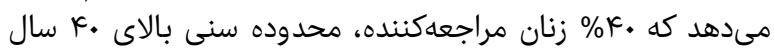

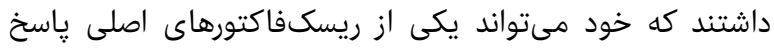

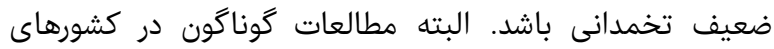

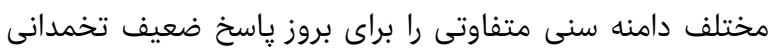

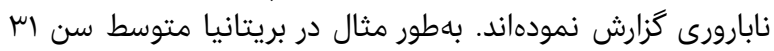

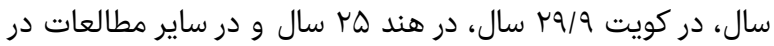

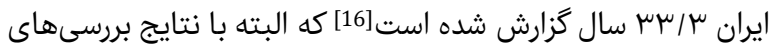

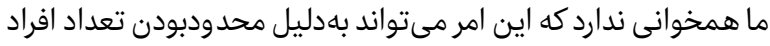

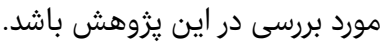

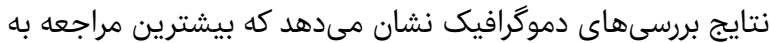

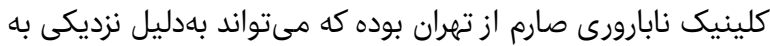

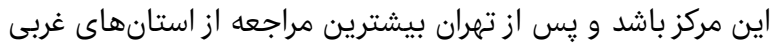

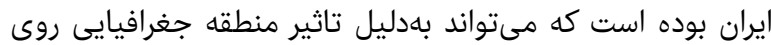

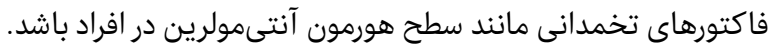

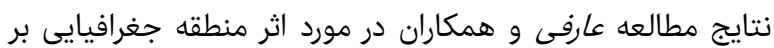

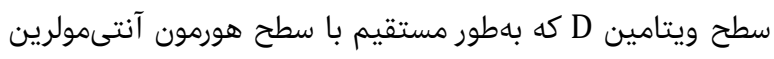

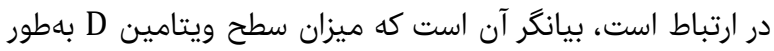

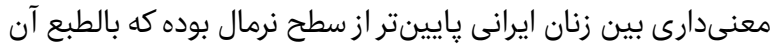

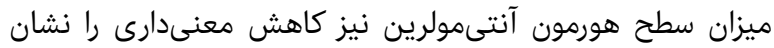

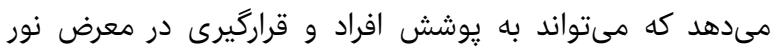

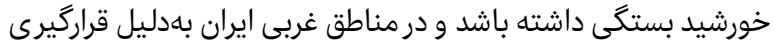

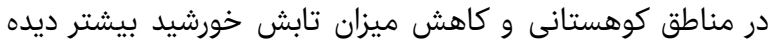

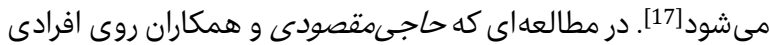
كه وارد يروسه ISCI مىشوند انجام دادند، به اين نتيجها 
Topcu 0 , et al. Obesity alters retrieved oocyte count and clinical pregnancy rates in high and poor responder women after in vitro fertilization. Arch Gynecol Obstet. 2010;282(1):89-96.

5- Garcia JE, Jones GS, Acosta AA, Wright G. Human menopausal gonadotropin/human chorionic gonadotropin follicular maturation for oocyte aspiration: Phase II, 1981. Fertil Steril. 1983;39(2):174-9.

6- Habibzadeh V, Ahmadi S. Evaluation of the results of ovulation induction with GnRH antagonist (Cetrotide) in patients with poor ovarian response in the previous ART cycle. Persian. Iran J Obstet Gynecol Infertility. 2015;18(155-156):1-8. [Persian]

7- Kaur M, Arora M. Diminished ovarian reserve, causes, assessment and management. Int J Infertility Fetal Med. 2013;4(2):45-55.

8- Ferraretti A, La Marca A, Fauser B, Tarlatzis B, Nargund G, Gianaroli L, et al. ESHRE consensus on the definition of 'poor response'to ovarian stimulation for in vitro fertilization: The bologna criteria. Hum Reprod. 2011;26(7):1616-24.

9- Schimberni M, Ciardo F, Schimberni M, Giallonardo A De Pratti V, Sbracia M. Short gonadotropin-releasing hormone agonist versus flexible antagonist versus clomiphene citrate regimens in poor responders undergoing in vitro fertilization: A randomized controlled trial. Eur Rev Med Pharmacol Sci. 2016;20(20):4354-61.

10- Ubaldi F, Vaiarelli A, D’Anna R, Rienzi L. Management of poor responders in IVF: Is there anything new. BioMed Res Int. 2014;2014:10.

11- Shrestha D, La X, Feng HL. Comparison of different stimulation protocols used in in vitro fertilization: A review. Ann Transl Med. 2015;3(10):137.

12- Kim CH. Strategies for poor responders in IVF cycles. Reproductive Syst Sexual Disord. 2011;5:001.

13- Patrizio P, Vaiarelli A, Setti PEL, Tobler KJ, Shoham G, Leong $\mathrm{M}$, et al. How to define, diagnose and treat poor responders? Responses from a worldwide survey of IVF clinics. Reproductive Biomed Online. 2015;30(6):581-92. 14- Alviggi C, Andersen CY, Buehler K, Conforti A, De Placido G, Esteves SC, et al. A new more detailed stratification of low responders to ovarian stimulation: from a poor ovarian response to a low prognosis concept. Fertil Steril. 2016;105(6):1452-3.

15- Humaidan P, Alviggi C, Fischer R, Esteves SC. The novel poseidon stratification of 'low prognosis patients in assisted reproductive technology' and its proposed marker of successful outcome. F1000Res. 2016;5:2911.

16- Hussein M, Alalaf S, Al-Tawil N. Effect of maternal age on the ovarian reserve markers, and pregnancy outcome in a sample of Kurdish women in Erbil city. Zanco J Med Sci. 2018;22(1):8-16.

17- Arefi S, Khalili G, Iranmanesh H, Farifteh F, Hosseini A, Fatemi HM, et al. Is the ovarian reserve influenced by vitamin $\mathrm{D}$ deficiency and the dress code in an infertile Iranian population?. J Ovarian Res. 2018;11(1):62.

18- Haji Maghsoudi F, Khalili M A, Aflatounian A. Complete oocyte maturation arrest in art cycles. J Reproduc Infertility. 2005;6(2):163-70. [Persian]

19- Mirza Moradi M, Sarefejoo FS, Bakhtiyari M, Mahmoodi M, Heidar Z. Relation between AMH level and ovarian reserve in infertile women candidate for assisted reproductive treatment. Med J Mashhad Univ Med Sci. 2017;60(4):597-609. [Persian]

20- Tolunay E. Treatment modalities in poor responder patients undergoing assisted reproductive techniques. Obstet Gynecol Int J. 2018;9(6):458-63.

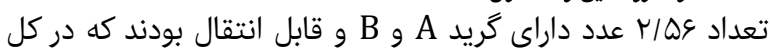

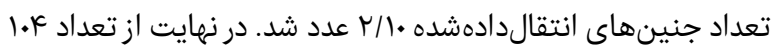

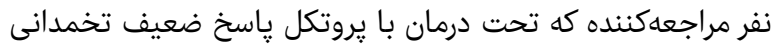

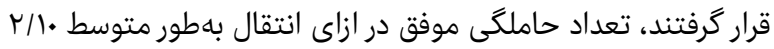

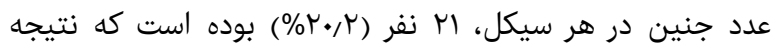

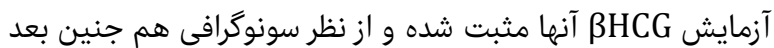

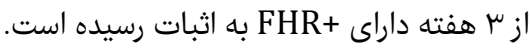

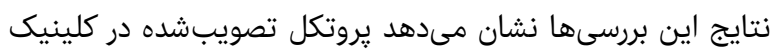

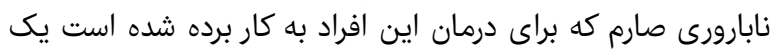

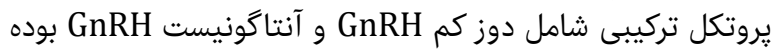

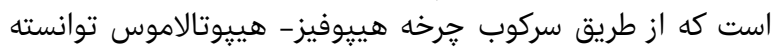

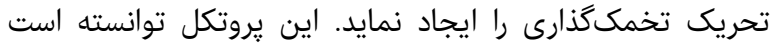

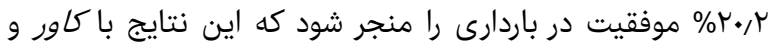

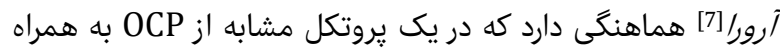

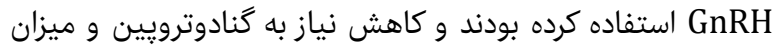

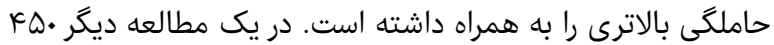

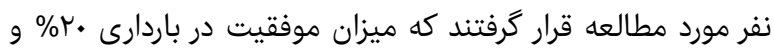

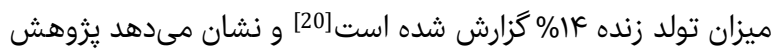

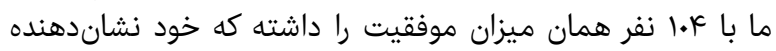

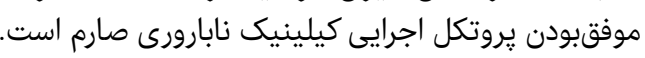

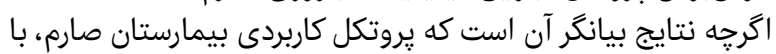

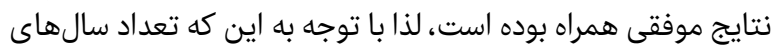

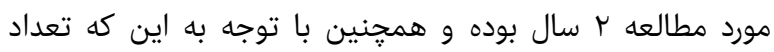

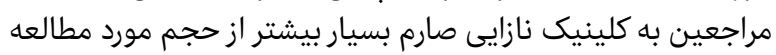

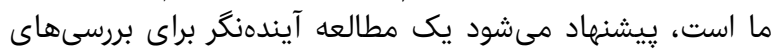

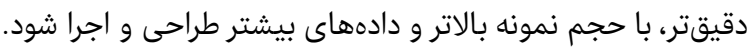

\section{نتيجهاگيرى}

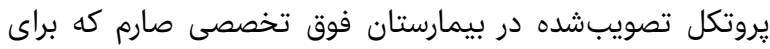

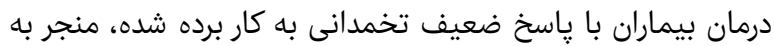

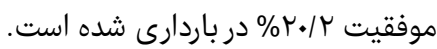

تشكر و قدردانى: از جناب آقاى دكتر صارمى و كاركنان محترم كلينيك

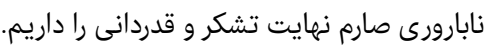

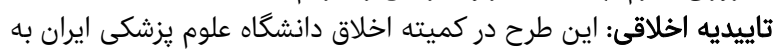

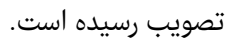
تعارض منافع: موردى از سوى نويسندكان كزارش نوار نشده است.

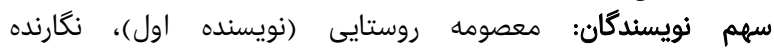

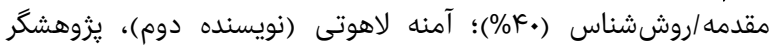

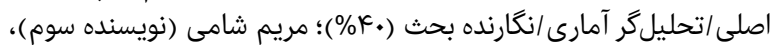

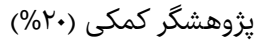
منابع مالى: توسط مركز تحقيقات بارورى و نابارورى صارم تامين شده است.

\section{منابع}

1- Gardner DK, Weissman A, Howles CM, Shoham Z. Textbook of assisted reproductive technologies: Laboratory and clinical perspectives. Boca Raton, Florida: CRC Press; 2009.

2- Jirge PR. Poor ovarian reserve. J Hum Reprod Sci. 2016;9(2):63-9.

3- Nichi M, de Cassia Sávio Figueira R, de Almeida Ferreira Braga DP, Setti AS, Iaconelli A, Borges E. Decreased fertility in poor responder women is not related to oocyte morphological status. Arch Med Sci. 2011;7(2):315-20.

4- Kilic S, Yilmaz N, Zülfikaroglu E, Sarıkaya E, Kose K, 